\title{
Editorial
}

J. Synchrotron Rad. (1999). 6, 809-811

\section{Synchrotron radiation and structural biology}

\author{
S. Samar Hasnain, ${ }^{a}$ John R. Helliwell ${ }^{b}$ and Hiromichi Kamitsubo ${ }^{c}$ \\ ${ }^{a}$ Synchrotron Radiation Department, CLRC Daresbury Laboratory, Warrington WA4 4AD, UK, \\ ${ }^{b}$ Department of Chemistry, Manchester University, Manchester M13 9PL, UK, and \\ 'SPring-8, JASRI, Kamigori-cho, Hyogo-ken 678-12, Japan
}

The motivation for this Special Issue has arisen from the award of the Nobel Prize in Chemistry (shared) in 1997 to Sir John Walker of the MRC's Laboratory of Molecular Biology [see J. Synchrotron Rad. (1998). 5, 1323]. This included the structure elucidation of the enzyme F1ATPase, which included extensive data collection at the SRS in Daresbury (Abrahams et al., 1994). This is then a chance to salute that work in the pages of this journal. A photograph of Sir John Walker's prize-winning team (Fig. 1) and a schematic representation of the structure of F1-ATPase (Fig. 2) are shown.

This is a further signal of the importance of synchrotron radiation in structural biology, which is seeing considerable

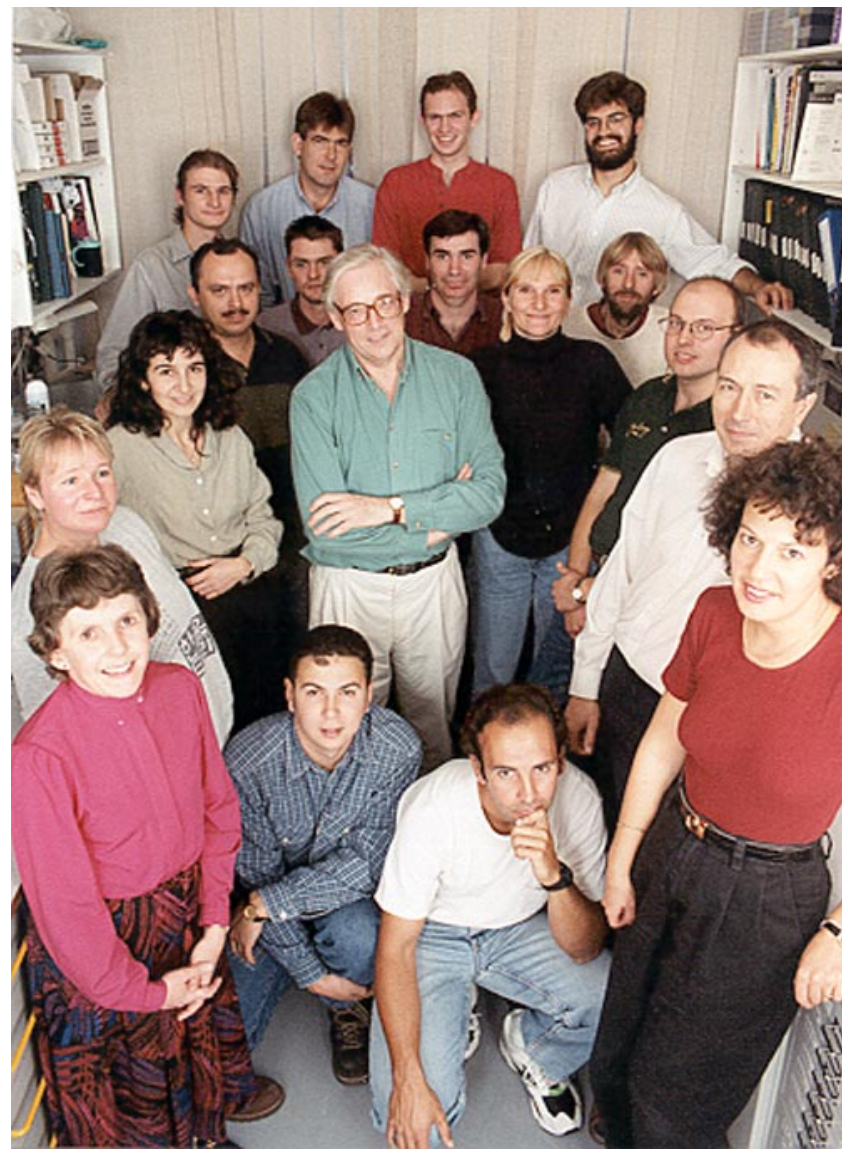

Figure 1

Sir John Walker and his team. growth. A range of techniques using synchrotron radiation are contributing: specifically protein crystallography, fibre diffraction, XAFS, small-angle scattering and microscopy. Other techniques are also growing in importance in the UV/VUV region of the spectrum. These include circular dichroism and time-resolved fluorescence utilizing the single-bunch mode of the synchrotron radiation operation.

In this special issue the Editorial Board of the Journal of Synchrotron Radiation (JSR) has commissioned various articles as a sample of the various aspects of synchrotronradiation-based structural biology. We are very grateful to all the authors for their articles. These have been fully refereed in the usual way. Also we are fortunate to have an overview article by Tom Blundell and Louise Johnson, who wrote one of the most popular monographs (Blundell \&

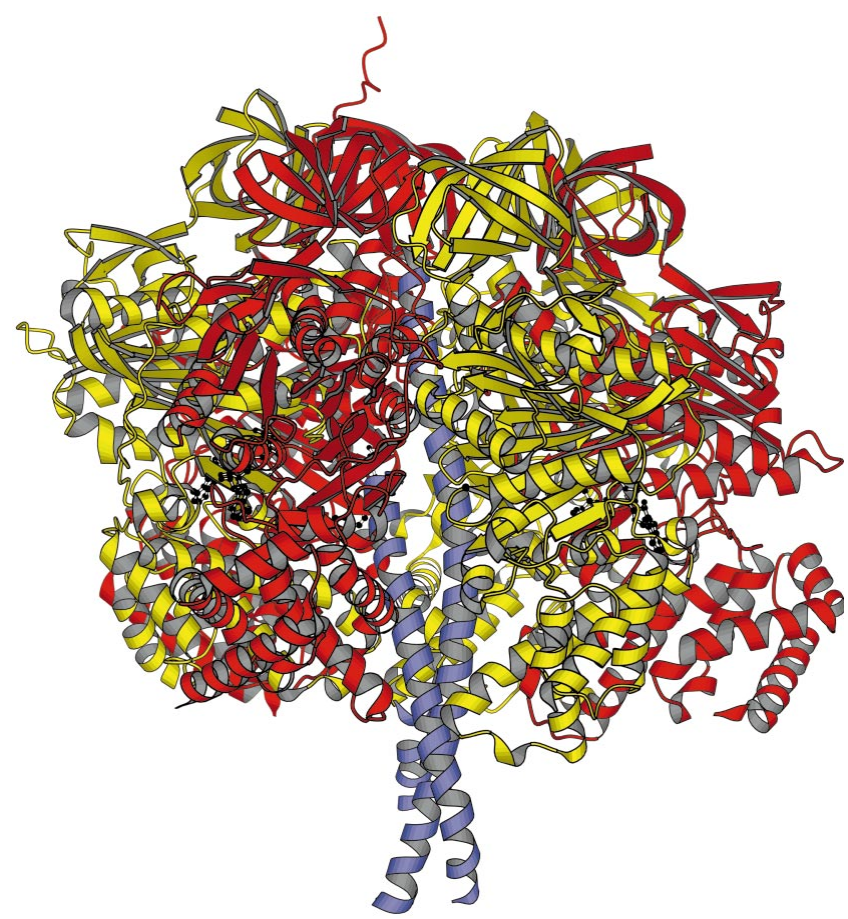

Figure 2

A schematic representation of the structure of bovine mitochondrial F1-ATPase determined at $2.8 \AA$ resolution using data collected at the SRS, Daresbury, UK, as reported by J. P. Abrahams, A. G. W. Leslie, R. Lutter and J. E. Walker [Nature (London) (1994), 371, 621-628]. 
Johnson, 1976) on protein crystallography at the time when synchrotron-radiation-based crystallography was in its infancy. Their overview provides a commentary on the articles in this issue and also looks at the future of synchrotron radiation and structural biology. Clearly we could have had a very large number of articles with many more authors and many more facilities represented. Some of those invited were not able to contribute due to prior commitment. The articles are written by leading authorities in the field and cover work carried out on DESY, Elettra, ESRF, LURE and SRS in Europe, Photon Factory and SPring-8 in Japan, and APS, CHESS, NSLS in the USA.

The transformation in structural biology brought together by synchrotron radiation is evident from the articles in this issue. On the one hand, atomic details of a blue tongue virus, a molecular assembly with a diameter of $700 \AA$, has become possible while, on the other hand, atomic-resolution structures and XAFS are providing information about the subtle aspects of the structure which control and regulate the function (e.g. catalysis, electron transfer) of a biomolecule. Synchrotron radiation has transformed protein crystallography and a current survey of the Protein Data Bank reveals that almost $55 \%$ of the deposited structures are determined using synchrotron radiation sources. The number of atomic resolution structures is increasing which currently number 128 . Further advances in synchrotron radiation sources and detectors together with crystallization and better data-collection methods are likely to make a significant impact in this area. MAD has become a very powerful method and is likely to increase in its importance with the increasing number of dedicated

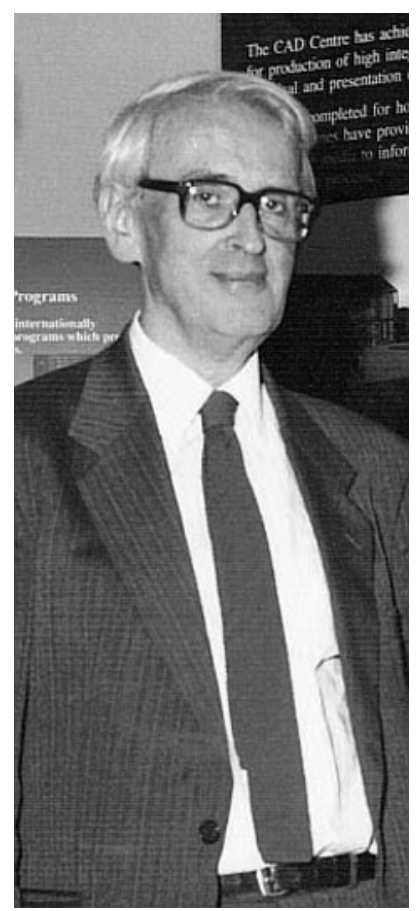

Figure 3

Lord Phillips of Ellesmere. experimental stations. The Laue method has come of age and has yielded a variety of time-resolved protein structural studies, reaching even the sub-nanosecond scale. Static and time-resolved X-ray fibre diffraction continue to benefit from the increased intensity of the third-generation sources. The central role of synchrotron radiation in biological and biomedical research was recently recognized in a European Science Foundation review (Hoff et al., 1998).

As another measure of the vigour of things to come, new synchrotron radiation sources with a strong structural biology component have been approved very recently in Canada and are at an advanced implementation stage in the UK. We include in this issue announcements recently made about these new synchrotron radiation sources.

Sadly, a leading figure in structural biology, Lord Phillips (Fig. 3), died during the preparation of this special issue. He was a leader in the founding of structural biology, especially through his crystallographic work on lysozyme and its catalytic mechanism (Fig. 4) and his seminal ideas on protein folding. He also made extensive contributions to the administration of science in the UK over many years.

Finally, we should state that our intention with this issue is forward looking. The historical antecedents of synchrotron radiation and structural biology were covered in the 'Fifty Years of SR' special issue of JSR in November 1997 (see e.g Doniach et al., 1997; Huxley \& Holmes, 1997, Munro, 1997) and have not been revisited here. As a signal of what is coming next, we mention now that a special issue of $J S R$ on spectroscopic imaging is in preparation. We commend this special issue for your interest and pleasure and urge you to make the Journal of Synchrotron Radiation an essential journal in your library.

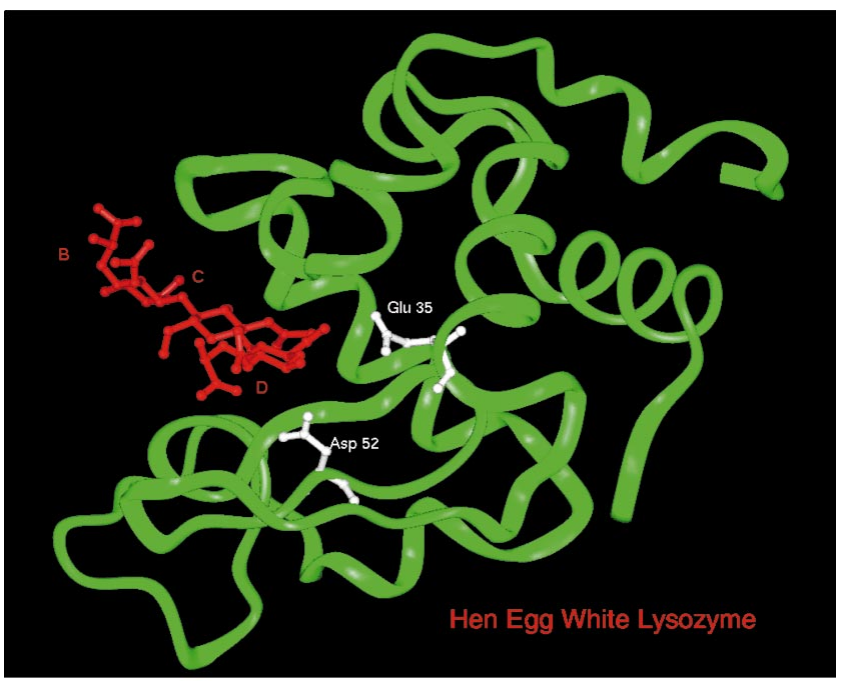

Figure 4

Lysozyme protein structure, the first enzyme whose structure was solved by X-ray crystallography by David Phillips and his team in the early- to mid-1960s (see Phillips, 1966). 


\section{References}

Abrahams, J. P., Leslie, A. G. W., Lutter, R. \& Walker, J. E. (1994). Nature (London), 371, 621-628.

Blundell, T. \& Johnson, L. N. (1976). Protein Crystallography. Oxford: Academic Press.

Doniach, S., Hodgson, K., Lindau, I., Pianetta, P. \& Winick, H. (1997). J. Synchrotron Rad. 4, 380-395.
Hoff, A., Lengeler, B., Perham, R. N., Öquist, G., Rest, M. V. \& Schirmer, T. (1998). ESF Studies on Large Research Facilities in Europe - Review of the Needs for European Synchrotron and Related Beamlines for Biological and Biomedical Research. ESF No. 983475.

Huxley, H. E. \& Holmes, K. C. (1997). J. Synchrotron Rad. 4, 366-379.

Munro, I. H. (1997). J. Synchrotron Rad. 4, 344-358.

Phillips, D. C. (1966). Sci. Am. 215(5), 78-90. 